\title{
The Need for Psychological Rehabilitation in Lung Transplant Recipients
}

Progress in Transplantation I-4

(C) 2020, NATCO. All rights reserved. Article reuse guidelines: sagepub.com/journals-permissions DOI: 10.1 I77/I526924820913510 journals.sagepub.com/home/pit (S)SAGE

\author{
Anahli Patel, PsyD' $\odot$, and Yelena Chernyak, PhD' ${ }^{\top}$
}

\begin{abstract}
Transplant recipients have significant psychosocial stressors due to unique posttransplant sequela that results in an increased incidence of psychopathology. Posttransplant psychological interventions, especially in lung transplant recipients, are understudied, as the focus of prior research has emphasized pretransplant interventions. However, posttransplant psychological stability affects medical outcomes. The importance of posttransplant psychological intervention is highlighted. Recommendations exist which call for attention to specific psychological domains in the posttransplant recovery period and highlight the impact of psychological rehabilitation on overall wellness and success in recovery. A novel psychological rehabilitation intervention is outlined as a response to posttransplant intervention recommendations to demonstrate implementation.
\end{abstract}

\section{Keywords}

lung transplant recipient, other transplant recipient, psychological rehabilitation, psychosocial intervention, behavioral disciplines and activities, education

\section{Introduction}

Previous studies have demonstrated that psychological stability improves over time in most transplant recipients alongside their medical status. ${ }^{1}$ Prevalence rates of anxiety and depression remain high in transplant recipients, mirroring those with chronic medical conditions. ${ }^{2}$ Research specific to lung transplant recipients is scarce and what is available may not be generalizable to this population. It has been established that of all transplant populations, lung transplant recipients have the highest rates of morbidity and mortality following transplantation $^{3}$ and are associated with higher levels of depression and psychological distress. ${ }^{4}$ The link between poor mental health and an increased risk of mortality, medical noncompliance, and medical complications has been well-documented in transplant recipients. ${ }^{1,3-5}$ While pretransplant evaluations for lung transplant patients are typically the focus of clinical and medical teams, posttransplant psychological factors may be just as important for success as psychosocial vulnerabilities can manifest as physical symptoms. ${ }^{5}$ Posttransplant psychological risk needs proactive consideration.

\section{Clinical Relevancy to Practice}

It stands to reason that psychological distress postsurgically may impact transplant outcomes both directly (eg, nonadherence with antirejection medications due to cognitive and motivational effects of depression) and indirectly (eg, less physical activity and socialization due to low mood which leads to deconditioning). The relationship between pretransplant psychosocial factors, including mental health, medical compliance, and substance use with posttransplant outcomes, has been established. ${ }^{6}$ The development of psychotherapeutic interventions following transplantation may further lessen the risk of complications and promote a sense of psychological wellness. Therefore, it is imperative to focus on post- and prepsychological wellness for optimized transplant outcomes. A paradigm that is well established in transplant programs is the concept of physical rehabilitation for optimal medical recovery, implemented routinely as a posttransplant best practice. $^{7}$ The model of physical rehabilitation could be extended to include parallel psychological rehabilitation programming for improving psychological and, consequently, medical outcomes.

A meta-analysis by Dew and colleagues ${ }^{1}$ identified key psychosocial themes associated with improved psychological and medical outcomes in organ transplant recipients. The 4 main domains include psychological (clinical disorders, cognitive functioning), behavioral (medical compliance, substance use/abuse), social (return to work, relationship stability, sexual

\footnotetext{
'Indiana University School of Medicine, IU Health Neurosciences Center/ Goodman Hall, Indianapolis, IN, USA
}

\section{Corresponding Author:}

Anahli Patel, Indiana University School of Medicine, IU Health Neurosciences Center/Goodman Hall, 355W, I6th St Suite 2800, Indianapolis, IN 46260, USA. Email: patelana@iu.edu 
activity, and social adjustment), and global quality of life (perceived health, effects of daily functioning, happiness, and satisfaction). Psychological skills with demonstrated benefit for lung transplant recipients include (1) mindfulness interventions to reduce distress and anxiety, ${ }^{8}(2)$ general coping strategies to improve mood, ${ }^{2}$ (3) social support and social adaption to returning to work/everyday life, ${ }^{9}$ and (4) discussions and support regarding body image, self-esteem, and sexual function. ${ }^{10}$ Each of these domains can directly affect a patient's medical status. If a patient experiences distress in one or more of these areas, their ability to successfully adhere to their medical needs may worsen. Consequently, these domains and skills should be addressed in transplant recovery.

\section{Practice Issues}

Posttransplant psychological support is an important, but overlooked, element in optimizing transplant outcomes, particularly in lung transplant recipients who have some of the highest rates of complications and distress following transplantation. ${ }^{4} \mathrm{~A}$ variety of barriers to establishing posttransplant support exist, which may contribute, in part, to why most transplant programs do not adequately address this need. These barriers may include a lack of trained personnel, poor patient willingness to engage, competing medical demands, cost, space, time, and distance. Since no recommendations yet exist in the literature on implementation, there is a lack of awareness and tools to adequately develop programming for enhanced posttransplant psychosocial recovery.

The literature has identified strategies to optimize psychological functioning in transplant recipients. ${ }^{2,8}$ The next step is to develop psychosocial interventions modeled as a form of psychological rehabilitation, delivered either concurrently or in parallel with physical rehabilitation. The rehabilitation model is based on a wellness model which assumes all transplant recipients will have something to gain from targeted intervention to improve emotional coping skills, reducing the risk of both overpathologizing psychological distress reactions to transplantation and omitting patients who appear to be doing well outwardly from needed psychological support. A group format may help address barriers to program implementation, such as such as cost, space, and limited personnel. Resources might also be shared between psychological and physical rehabilitation programs to leverage increased buy-in, promote communication between specialties, foster community and socialization, and utilize valuable clinic resources by addressing them in a group format.

\section{Implementation of a Psychological Rehabilitation Program for Lung Transplant Recipients}

As a part of the post-lung transplant rehabilitation program, recipients attend a unique hospital-based daily physical rehabilitation service, into which a weekly 1-hour long psychological rehabilitation program is incorporated to address psychological domains associated with improved transplant outcomes. This novel group therapy program, titled "Cardiothoracic Patients Linking Mind and Body" (CLIMB), focuses on stress management, mindfulness, optimizing mood, and communication skills derived from the literature. Table 1 outlines each module of CLIMB. This group therapy program works in sync with the goals of physical rehabilitation by promoting wellness skills, strengthening motivation, encouraging medical adherence, and providing skills and opportunities for social adaptation after transplant. The program functions as an open group, since patients start in tandem with the physical rehabilitation program and continue for its duration. Average participation is 4 weeks. The sessions of CLIMB continuously rotate so that regardless of when a patient enters the rehabilitation center, they will attend, on average, all 4 weekly sessions over the course of their rehabilitation. The group is led by the transplant psychologist who is part of the transplant team in a private space located in the rehabilitation center. Patient census varies, ranging from 2 to 9 patients. Some patients may require further intervention in conjunction with or following the CLIMB program, including outpatient psychiatry consultations or ongoing individual psychotherapy provided by transplant psychology or in the community. This is determined by the transplant psychologist on a case-by-case basis with input from the rest of the medical team.

The first module on managing stress walks patients through the steps to build a coping skills toolbox, an inventory of different strategies available for emotional distress management. The importance of relaxation as a coping skill is introduced. The second module focuses on mood and understanding how thoughts, feelings, and behaviors impact each other. The coping skills discussed here help identify and improve negative mood or depression symptoms. If symptoms of depression can be reduced, physical well-being improves. ${ }^{5}$ The third module discusses how to express emotions constructively, fostering acceptance of changing life situations, identifying and communicating needs to others, and gathering and/or changing social support structures posttransplant. Many patients identify changes to their psychosocial functioning as most distressing even after health status improves. Lastly, the fourth module emphasizes creating achievable and attainable goals, managing pitfalls or setbacks in any domain of life, and understanding the cycle of change which can help in creating and maintaining life changes. Each module culminates with a meditation or relaxation exercise to further solidify and practice skills that have been shown to decrease anxiety and depression symptoms. ${ }^{8}$

Limitations to psychological rehabilitation programs such as CLIMB include their brief nature. In fact, a sample of anonymous satisfaction surveys completed at program completion has indicated a preference for increased session frequency. Further, CLIMB is one example of a posttransplant intervention. Ongoing research is necessary to ascertain what type of psychosocial intervention is most beneficial for improving transplant outcomes. Consideration for patient preferences and logistical challenges are required to implement any additional intervention in the posttransplant period, as resources, time, 
Table I. Program Curriculum for Postlung Transplantation Psychological Rehabilitation.

CLIMB: Cardiothoracic Patients Linking Mind and Body

Program Curriculum

Module I Adrenaline rush: Sustaining emotional wellness during times of stress

- Deconstructing stress into 2 areas: distress (negative stress) and eustress (positive stress)

- Psychoeducation about "fight/flight/freeze" responses to stress: how stress and worry can impact wellness

- Creating a stress inventory: identifying sources and signs of stress, including physical, emotional, psychological, and behavioral symptoms

- Developing a personalized skill and coping toolbox: skills to manage symptoms as they arise

- Managing physical impacts of stress: introduction to relaxation, mindfulness, and meditation techniques

- End with diaphragmatic breathing exercises

Module 2 Mood boosters: Cultivating a healthy attitude in the face of adversity

- Cognitive triad: how thoughts, feelings, and behaviors impact our mood

- How to disrupt the cognitive triad

- Thoughts: identifying negative thinking styles and patterns, noticing the "inner critic" and positive self-talk, challenging negative thought

- Feelings: identifying and describing emotions associated with mood

- Behaviors: identifying pleasurable activities, natural mood boosters, and getting (re)engaged in life

- Self-care: healthy eating and sleep

- End with a progressive muscle relaxation exercise

Module 3 People matter: Improving communication and relationships in the face of transplant

- Psychoeducation on how social relationships before and after transplant can change: a visual representation is created using bull's-eye model with patient at center, and each level a different person or social group (ie, medical team, caregivers, other family, coworkers/friends, etc)

- Identifying changes in needs: how pre- and posttransplant needs change and what psychosocial changes are occurring

- How to express emotions and needs constructively: discuss communication styles and how to engage in assertive communication

- Fostering acceptance

- End with a guided imagery exercise

Module 4 Motivation and acceptance: Staying the course after transplant

- Overview of the CLIMB Program Wellness Inventory: addressing dimensions of wellness including physical, emotional, intellectual, interpersonal, spiritual, and environmental

- Discussion of behaviors associated with self-care: medical compliance, accountability, motivators, and reinforcement

- Managing pitfalls: discussion of the cycle of change, creating SMART goals (specific, measureable, attainable, realistic, timely) to create and maintain changes

- Resources and discussions for adaptive living: topics can include insurance, disability/work, transportation, legal, psychological support, and so on, which are driven by patient participation

- End with guided meditation/mindfulness exercise

and energy are at a premium during this critical recovery period. Our program has initiated collection of self-report measures evaluating levels of and changes to anxiety, depression, and distress symptoms during the CLIMB program, which shows early signs of symptom improvement during this treatment phase. Future projects must further evaluate both longand short-term outcomes of implementing posttransplant psychosocial support, with possible impact on transplant outcomes, morbidity, mortality, and medical adherence, while controlling for the effects of standard transplant recovery.

\section{Summary and Implications for Practice}

Physical and health-related aspects of a patient's health status improve after transplant, but psychological and emotional health of patients may continue to be negatively affected. Research has initiated a call for action as psychosocial support posttransplant has been deemed necessary ${ }^{1,2,4,10}$ but has not been widely implemented in clinical practice. Although lung transplant recipients have better general, physical, and psychological health as compared to their pretransplant counterparts, they still experience a lower quality of life overall. ${ }^{10}$ More recent literature is starting to examine the nuances of the relationship between posttransplant psychosocial functioning and survival, incidents of medical complications, and noncompliance. The connection between posttransplant success has been directly related and established with long-term survival. ${ }^{4}$ Future directions related to CLIMB include the need to implement and assess program outcomes. Psychological rehabilitation posttransplant may be an effective intervention to promote psychological wellness in the short and long term after a taxing and complicated medical procedure such as lung transplantation to improve the likelihood of success and increase quality of life.

\section{Authors' Note}

Consent obtained as part of clinical protocol. 


\section{Declaration of Conflicting Interests}

The author(s) declared no potential conflicts of interest with respect to the research, authorship, and/or publication of this article.

\section{Funding}

The author(s) received no financial support for the research, authorship, and/or publication of this article.

\section{ORCID iD}

Anahli Patel, PsyD (D) https://orcid.org/0000-0003-3894-7032

Yelena Chernyak, PhD (D) https://orcid.org/0000-0001-6925-1981

\section{References}

1. Dew MA, Switzer GE, DiMartini AF, Matukaitis J, Fitzgerald MG, Kormos RL. Psychosocial assessments and outcomes in organ transplantation. Prog Transplant. 2000;10(4):239-259.

2. Dew MA, DiMartini AF. Psychological disorders and distress after adult cardiothoracic transplantation. J Cardiovasc Nurs. 2005;20(5 suppl):S51-S66.

3. Rosenberger EM, DiMartini AF, DeVito Dabbs AJ, et al. Psychiatric predictors of long-term transplant-related outcomes in lung transplant recipients. Transplantation. 2016;100(1): 239-247. doi:10.1097/TP.0000000000000824.
4. Smith PJ, Blumenthal JA, Trulock EP, et al. Psychosocial predictors of mortality following lung transplantation. Am J Transplant. 2016;16(1):271-277. doi:10.1111/ajt.13447.

5. DeVito Dabbs AJ, Dew AM, Stilley CS, et al. Psychosocial vulnerability, physical symptoms and physical impairment after lung and heart-lung transplantation. J Heart Lung Transplant. 2003;22(11):1268-1275.

6. Maldonado JR, Sher Y, Lolak S, et al. The Stanford integrated psychosocial assessment for transplantation: a prospective study of medical and psychosocial outcomes. Psychosom Med. 2015; 77(9):1018-1030. doi:10.1097/PSY.0000000000000241.

7. Nici L, Donner C, Wouters E, et al. American Thoracic Society/ European Respiratory Society statement on pulmonary rehabilitation. Am J Respir Crit Care Med. 2006;173(12):1390-1413.

8. Gross CR, Kreitzer MJ, Russas V, Treesak C, Frazier PA, Hertz MI. Mindfulness mediation to reduce symptoms after organ transplant: a pilot study. Adv Mind Body Med. 2004;20(2):20-29.

9. Paris W, White-Williams C. Social adaptation after cardiothoracic transplantation: a review of the literature. $J$ Cardiovasc Nurs. 2005; 20(5 suppl):S67-S73.

10. Limbos MM, Joyce DP, Chan CK, Kesten S. Psychological functioning and quality of life in lung transplant candidates and recipients. Chest. 2000;118(2):408-416. 